\title{
Effect of Nitrogen and Weed Management on Nutrient Removal by Weed in Direct Seeded Rice
}

\author{
B. Rama Devi* and Yashwant Singh \\ Department of Agronomy, Institute of Agricultural Sciences, BHU, Varanasi, India \\ *Corresponding author
}

\begin{tabular}{|c|}
\hline Keywords \\
\hline $\begin{array}{l}\text { Direct seeded rice, } \\
\text { Nutrient removal, } \\
\text { weed management, } \\
\text { weed drymatter }\end{array}$ \\
\hline Article Info \\
\hline $\begin{array}{l}\text { Accepted: } \\
16 \text { May } 2018 \\
\text { Available Online: } \\
10 \text { June } 2018\end{array}$ \\
\hline
\end{tabular}

A B S T R A C T

A study was carried out during Kharif 2015 and 2016 to evaluate the effect of four nitrogen and six weed management practices on drymatter and nutrient removal by weed in direct seeded rice on a sandy loam soil at the Research Farm of Institute of Agricultural Sciences, Banaras Hindu University, Varanasi. The experiment was laid out in split plot design with 3 replications. The results revealed that the application of $1 / 4 \mathrm{~N}$ basal $+1 / 4 \mathrm{~N}$ at active tillering stage $+1 / 4 \mathrm{~N}$ at panicle initiation stage $+1 / 4 \mathrm{~N}$ at heading stage was significantly superior in reducing total weed dry matter $\left(\mathrm{g} \mathrm{m}^{-2}\right)$ at all the stages of observation and NPK removal by weeds at 60 DAS and at harvest than $1 / 2 \mathrm{~N}$ basal $+1 / 4 \mathrm{~N}$ at active tillering stage $+1 / 4 \mathrm{~N}$ at panicle initiation stage and $1 / 4 \mathrm{~N}$ at basal $+1 / 2 \mathrm{~N}$ at active tillering stage $+1 / 4 \mathrm{~N}$ at panicle initiation stage. However, it was at par with $1 / 3 \mathrm{~N}$ at basal $+1 / 3 \mathrm{~N}$ at active tillering stage $+1 / 3 \mathrm{~N}$ at panicle initiation stage. Amongst weed management practices hand weeding twice at 20 and 40 DAS followed by bispyribac at 25 g a.i. $\mathrm{ha}^{-1}+$ azimsulfuron at $17.5 \mathrm{~g}$ a.i. $\mathrm{ha}^{-1}+$ NIS $(0.25 \%)$ at $15-20$ DAS proved most effective in reducing weed drymatter and nutrient removal by weeds as compared to other herbicidal treatments. Application of oxadiargyl at $90 \mathrm{~g}$ a.i. $\mathrm{ha}^{-1}(\mathrm{PE}) \mathrm{fb}$ bispyribac at $25 \mathrm{~g}$ a.i. ha ${ }^{-1}+$ NIS $(0.25 \%)$ at $15-20$ DAS had minimum efficacy in these respect during both the years.

\section{Introduction}

In recent years there has been a shift from transplanting to direct seeding. This shift was principally driven by water scarcity issues and expensive labour component for transplanting under acute farm labour shortage (Chauhan, 2012). Direct seeding of rice has the potential to provide several benefits to farmers and the environment over conventional practices of puddling and transplanting. Direct seeding helps reduce water consumption by about $30 \%$ (0.9 million liters acre -1) as it eliminates raising of seedlings in a nursery, puddling, transplanting under puddled soil and maintaining 4-5 inches of water at the base of the transplanted seedlings (Joshi et al., 2013). Despite several advantages of DSR, it is subjected to much higher weed pressure than the conventional puddled transplanted rice systems (Chauhan, 2012), in which weeds are suppressed by standing water and transplanted rice seedlings. Aerobic soil conditions and alternate wetting and drying in DSR are conducive to the germination and growth of weeds, causing grain yield losses of up to 
$80 \%$. Thus, an efficient and timely weed control is crucial for the success of DSR. In order to control weeds, farmers use both pre and post emergence herbicides (Mahajan et al., 2013).Weeds interfere with normal crop growth by competing for available nutrients, light and water. Uncontrolled weeds reduce the grain yield by $96 \%$ in dry direct-seeded rice and $61 \%$ in wet direct seeded rice (Maity and Mukerjee, 2008). Nitrogen is a key nutrient in determining the level of crop productivity. The efficiency of applied nitrogen is very low and varies from 20 to $25 \%$ in upland rice crop due to the oxidized condition prevailing in uplands and concomitant heavy nitrogen loss through percolating water. Hence, fractional application of nitrogen in right amount and proportion, and when it is needed the most seems to be a practical proposition. Weed control also facilitates higher absorption of applied nutrient, thus increases the efficiency of fertilizers application to the crops (Amarjit et al., 2006). Split application is one of strategies for efficient use of $\mathrm{N}$ fertilizers throughout the growing season by synchronizing with plant demand, reducing denitrification losses and improved $\mathrm{N}$ uptake by crop.

\section{Materials and Methods}

A field experiment was conducted during rainy (kharif) season of 2015 and 2016 at Agricultural Research Farm, Department of Agronomy, Institute of Agricultural sciences, Banaras Hindu University, Varanasi, Uttar Pradesh. The soil was Gangetic alluvial having Sandy clay loam in texture with $\mathrm{pH}$ 7.60. It was moderately fertile, being low in available organic carbon $(0.40 \%)$, available $\mathrm{N}$ (198.38 $\left.\mathrm{kgha}^{-1}\right)$, and medium in available $\mathrm{P}$ $\left(17.78 \mathrm{~kg} \mathrm{ha}^{-1}\right)$ and $\mathrm{K}\left(216.32 \mathrm{~kg} \mathrm{ha}^{-1}\right)$. The experiment was laid out in split-plot design with three replications. The nitrogen management subjected to main plots while weed management in sub plots. A combination of 24 treatments consisting of 4 nitrogen management, viz. $\mathrm{N}_{1}-1 / 2 \mathrm{~N}$ basal $+1 / 4$ $\mathrm{N}$ at active tillering stage $+1 / 4 \mathrm{~N}$ at panicle initiation stage, $\mathrm{N}_{2}-1 / 4 \mathrm{~N}$ at basal $+1 / 2 \mathrm{~N}$ at active tillering stage $+1 / 4 \mathrm{~N}$ at panicle initiation stage, $\mathrm{N}_{3}-1 / 3 \mathrm{~N}$ at basal $+1 / 3 \mathrm{~N}$ at active tillering stage $+1 / 3 \mathrm{~N}$ at panicle initiation stage and $\mathrm{N}_{4}-1 / 4 \mathrm{~N}$ basal $+1 / 4 \mathrm{~N}$ at active tillering stage $+1 / 4 \mathrm{~N}$ at panicle initiation stage $+1 / 4 \mathrm{~N}$ at heading stage and 6 weed management treatments, viz. $\mathrm{W}_{0}$ Weedy check, $\mathrm{W}_{1}$ - Two hand weedings at 20 and 40 DAS, $W_{2}$ - Pendimethalin $1.0 \mathrm{~kg}$ a.i ha ${ }^{-}$ ${ }^{1}$ (PE) fb Bispyribac at $25 \mathrm{~g}$ a.i ha ${ }^{-1}+$ NIS $(0.25 \%)$ at $15-20 \mathrm{DAS}, \mathrm{W}_{3}-$ Bispyribac at 25 g a.i. $\mathrm{ha}^{-1}+$ Pyrazosulfuron at $20 \mathrm{~g}$ a.i. $\mathrm{ha}^{-1}+$ NIS $(0.25 \%)$ at $15-20$ DAS, $\mathrm{W}_{4}$ - Oxadiargyl at $90 \mathrm{~g}$ a.i. ha ${ }^{-1}$ (PE) $f b$ Bispyribac at $25 \mathrm{~g}$ a.i. $\mathrm{ha}^{-1}+\mathrm{NIS}(0.25 \%)$ at $15-20$ DAS and $\mathrm{W}_{5}$ Bispyribac at $25 \mathrm{~g}$ a.i. ha $^{-1}+$ Azimsulfuron at $17.5 \mathrm{~g}$ a.i. $\left.\mathrm{ha}^{-1}\right)+\mathrm{NIS}(0.25 \%)$ at 15-20 DAS. A uniform dose of $150 \mathrm{~kg} \mathrm{~N}^{-1}, 60 \mathrm{~kg} \mathrm{P}_{2} \mathrm{O}_{5}$ $\mathrm{ha}^{-1}$ and $60 \mathrm{~kg} \mathrm{~K}_{2} \mathrm{O} \mathrm{ha}^{-1}$ were applied in all the plots.

Full dose of phosphorus and potash were applied as basal application and nitrogen was applied as treatment wise. 'HUR 105' variety of rice@ $35 \mathrm{~kg} \mathrm{ha}^{-1}$ was used for seeding of rice. The required quantity of pre-emergence and post-emergence herbicides was sprayed as per treatment using spray volume of 600 litres of water $\mathrm{ha}^{-1}$ with the help of knap sack sprayer fitted with flat fan nozzle.

\section{Results and Discussion}

Grassy weeds were predominant in DSR followed by sedges and broad leaved weeds, respectively. The dominant weed species observed in the experimental field were Echinocloa crus-galli, Echinocloa colona, Cynodon dactylon, Cyperus rotundus, Cyperus iria, Eclipta alba and Caesulia axillaris during both the years of study. 


\section{Effect on weed dry matter $\left(\mathrm{g} \mathrm{m}^{-2}\right)$}

Data clearly revealed that among nitrogen treatments, significantly lowest of total dry matter of weeds was observed with application of $1 / 4 \mathrm{~N}$ at basal $+1 / 4 \mathrm{~N}$ at active tillering stage $+1 / 4 \mathrm{~N}$ at panicle initiation stage $+1 / 4$ at heading stage which was at par to $1 / 3 \mathrm{~N}$ at basal $+1 / 3$ $\mathrm{N}$ at active tillering stage $+1 / 3 \mathrm{~N}$ at panicle initiation stage at all stage of observation during both the years of study. Whereas, maximum weed dry weight was recorded when nitrogen was applied as $1 / 4 \mathrm{~N}$ at basal + $1 / 2 \mathrm{~N}$ at active tillering stage $+1 / 4$ at panicle initiation stage. Application of $1 / 4 \mathrm{~N}$ at basal + $1 / 4$ at active tillering stage $+1 / 4 \mathrm{~N}$ at panicle initiation stage $+1 / 4$ at heading stage was the most effective treatment among all the nitrogen treatments in minimizing dry matter of weeds at all the stage of observation during both the years of experimentation. The higher dry weight of weeds under splits involving more nitrogen application at sowing might be attributed to vigorous growth of weeds due to excess nitrogen supply at sowing, which consequently resulted in higher dry weight of weeds at different stages of growth. Similar findings were given by Sanusan et al., (2010).

Among weed management practices, application of bispyribac at $25 \mathrm{~g}$ a.i. $\mathrm{ha}^{-1}+$ azimsulfuron at $17.5 \mathrm{~g}$ a.i. $\mathrm{ha}^{-1}+$ NIS $(0.25 \%)$ at 15-20 DAS was most effective in minimizing the total weed dry matter at all the crop growth stages and was found significantly superior to all the weed management practices next to the treatment two hand weedings at 20 and 40 DAS. Application of bispyribac at $25 \mathrm{~g}$ a.i. $\mathrm{ha}^{-1}+$ pyrazosulfuron at $20 \mathrm{~g}$ a.i. $\mathrm{ha}^{-1}+$ NIS $(0.25 \%)$ at 15-20 DAS was the next best treatment to minimise the weed dry matter and found superior to other treatments at all the stages during both the years. Both these weed management treatments were better than other weed management practices in minimizing dry weight of weeds in both the years of experimentation. Weedy check resulted in maximum weed dry matter at all the stages of crop growth during both the years. Tank mix application of azimsulfuron with bispyribacsodium effectively reduced the dry biomass of Cyperus spp. These findings may be supported by Ghosh et al., (2017).

\section{Effect on nutrient ( $N, P, K)$ removal by weeds $\left(\mathrm{Kg} \mathrm{ha}^{-1}\right)$}

\section{Nitrogen removal $\left(\mathrm{Kg} \mathrm{ha}^{-1}\right)$}

Nitrogen treatments differed significantly in nitrogen removal by weeds during both the years at 60 DAS and at harvest stage of direct seeded rice. The minimum nitrogen removal was recorded by application of $1 / 4 \mathrm{~N}$ at basal + $1 / 4$ at active tillering stage $+1 / 4 \mathrm{~N}$ at panicle initiation stage $+1 / 4$ at heading stage which was significantly less than all other nitrogen treatments except the application of $1 / 3 \mathrm{~N}$ at basal $+1 / 3 \mathrm{~N}$ at active tillering stage $+1 / 3 \mathrm{~N}$ at panicle initiation stage. These two treatments were at par with each other. Data also showed that nitrogen application of $1 / 4 \mathrm{~N}$ at basal $+1 / 2 \mathrm{~N}$ at active tillering stage $+1 / 4 \mathrm{~N}$ at panicle initiation stage had maximum nitrogen removal by weeds during both the years of study at 60 DAS and at harvest stage of direct seeded rice. This is in agreement with the findings of Sweeney et al., 2008. Among weed management practices, application of bispyribac at $25 \mathrm{~g}$ a.i. $\mathrm{ha}^{-1}+$ azimsulfuron at $17.5 \mathrm{~g}$ a.i. $\mathrm{ha}^{-1}+$ NIS $(0.25 \%)$ at 15-20 DAS established their superiority in minimizing the nitrogen removal by weeds which was significantly superior to other weed management treatments but it was next best to the two hand weedings at 20 and 40 DAS. Bispyribac at $25 \mathrm{~g}$ a.i. $\mathrm{ha}^{-1}+$ pyrazosulfuron at $20 \mathrm{~g}$ a.i. $\mathrm{ha}^{-1}+$ NIS $(0.25 \%)$ at $15-20$ DAS was the next best treatment in minimizing the removal of nitrogen by weeds during both the years at both the stages of crop growth. 
Table.1 Effect of nitrogen management and weed management practices on total weed dry matter $\left(\mathrm{g} . \mathrm{m}^{-2}\right)$ at different growth stages of direct seeded rice

\begin{tabular}{|c|c|c|c|c|c|c|c|c|c|c|}
\hline \multirow[t]{2}{*}{ Treatments } & \multicolumn{2}{|c|}{15 DAS } & \multicolumn{2}{|c|}{30 DAS } & \multicolumn{2}{|c|}{60 DAS } & \multicolumn{2}{|c|}{90 DAS } & \multicolumn{2}{|c|}{ Harvest } \\
\hline & 2015 & 2016 & 2015 & 2016 & 2015 & 2016 & 2015 & 2016 & 2015 & 2016 \\
\hline \multicolumn{11}{|l|}{ Nitrogen management } \\
\hline $\begin{array}{l}N_{1}-1 / 2 \text { at basal }+1 / 4 \text { at active tillering stage }+1 / 4 \mathrm{~N} \text { at } \\
\text { panicle initiation stage }\end{array}$ & $\begin{array}{c}5.34 \\
(28.38)\end{array}$ & $\begin{array}{c}5.73 \\
(32.64)\end{array}$ & $\begin{array}{c}6.57 \\
(44.27)\end{array}$ & $\begin{array}{c}7.38 \\
(55.74)\end{array}$ & $\begin{array}{c}8.48 \\
(72.23)\end{array}$ & $\begin{array}{c}9.18 \\
(84.84)\end{array}$ & $\begin{array}{c}9.99 \\
(108.28)\end{array}$ & $\begin{array}{c}11.34 \\
(129.30)\end{array}$ & $\begin{array}{c}9.83 \\
(87.65)\end{array}$ & $\begin{array}{c}10.83 \\
(118.07)\end{array}$ \\
\hline $\begin{array}{l}N_{2^{-}}-1 / 4 \mathrm{~N} \text { at basal }+1 / 2 \mathrm{~N} \text { at active tillering stage }+1 / 4 \\
\text { at panicle initiation stage }\end{array}$ & $\begin{array}{c}5.53 \\
(30.50)\end{array}$ & $\begin{array}{c}5.92 \\
(35.03)\end{array}$ & $\begin{array}{c}6.87 \\
(48.27)\end{array}$ & $\begin{array}{c}7.75 \\
(61.34)\end{array}$ & $\begin{array}{c}8.79 \\
(77.60)\end{array}$ & $\begin{array}{c}9.51 \\
(91.10)\end{array}$ & $\begin{array}{c}10.40 \\
(115.58)\end{array}$ & $\begin{array}{c}11.82 \\
(139.41)\end{array}$ & $\begin{array}{c}10.17 \\
(104.02)\end{array}$ & $\begin{array}{c}11.20 \\
(126.46)\end{array}$ \\
\hline $\begin{array}{l}N_{3^{-}} 1 / 3 \mathrm{~N} \text { at basal }+1 / 3 \mathrm{~N} \text { at active tillering stage }+ \\
1 / 3 \mathrm{n} \text { at panicle initiation stage }\end{array}$ & $\begin{array}{c}5.15 \\
(26.46)\end{array}$ & $\begin{array}{c}5.53 \\
(30.47)\end{array}$ & $\begin{array}{c}6.31 \\
(40.89)\end{array}$ & $\begin{array}{c}7.06 \\
(51.11)\end{array}$ & $\begin{array}{c}8.17 \\
(67.11)\end{array}$ & $\begin{array}{c}8.88 \\
(79.37)\end{array}$ & $\begin{array}{c}10.74 \\
(100.39)\end{array}$ & $\begin{array}{c}10.90 \\
(119.71)\end{array}$ & $\begin{array}{c}9.49 \\
(90.67)\end{array}$ & $\begin{array}{c}10.52 \\
(110.53)\end{array}$ \\
\hline $\begin{array}{l}\mathbf{N}_{4^{-}} 1 / 4 \mathbf{N} \text { at basal }+1 / 4 \text { at active tillering stage }+1 / 4 \mathbf{N} \\
\text { at panicle initiation stage }+1 / 4 \text { at heading stage }\end{array}$ & $\begin{array}{c}5.07 \\
(25.42)\end{array}$ & $\begin{array}{c}5.39 \\
(28.86)\end{array}$ & $\begin{array}{c}6.14 \\
(38.59)\end{array}$ & $\begin{array}{c}6.82 \\
(47.62)\end{array}$ & $\begin{array}{c}8.00 \\
(64.32)\end{array}$ & $\begin{array}{c}8.71 \\
(76.42)\end{array}$ & $\begin{array}{c}9.81 \\
(96.71)\end{array}$ & $\begin{array}{c}10.58 \\
(112.93)\end{array}$ & $\begin{array}{c}9.36 \\
(87.97)\end{array}$ & $\begin{array}{c}10.28 \\
(106.30)\end{array}$ \\
\hline SEm \pm & 0.059 & 0.057 & 0.076 & 0.074 & 0.090 & 0.090 & 0.102 & 0.154 & 0.105 & 0.112 \\
\hline $\mathrm{CD}(\mathrm{P}=\mathbf{0 . 0 5})$ & 0.203 & 0.197 & 0.263 & 0.255 & 0.310 & 0.311 & 0.354 & 0.533 & 0.365 & 0.387 \\
\hline \multicolumn{11}{|l|}{ Weed management practices } \\
\hline $\mathrm{W}_{0}$ - Weedy check & $\begin{array}{c}6.10 \\
(36.92)\end{array}$ & $\begin{array}{c}6.56 \\
(42.75)\end{array}$ & $\begin{array}{c}9.01 \\
(80.97)\end{array}$ & $\begin{array}{c}9.94 \\
(98.56)\end{array}$ & $\begin{array}{c}10.14 \\
(102.49)\end{array}$ & $\begin{array}{c}11.04 \\
(118.77)\end{array}$ & $\begin{array}{c}12.02 \\
(144.12)\end{array}$ & $\begin{array}{c}13.43 \\
(167.16)\end{array}$ & $\begin{array}{c}11.65 \\
(135.38)\end{array}$ & $\begin{array}{c}12.84 \\
(160.83)\end{array}$ \\
\hline $\mathrm{W}_{1^{-}}$Two hand weedings at 20 and 40 DAS & $\begin{array}{c}5.95 \\
(34.75)\end{array}$ & $\begin{array}{c}6.27 \\
(38.91)\end{array}$ & $\begin{array}{c}5.19 \\
(26.50)\end{array}$ & $\begin{array}{c}5.89 \\
(34.25)\end{array}$ & $\begin{array}{c}7.36 \\
(53.75)\end{array}$ & $\begin{array}{c}7.79 \\
(63.25)\end{array}$ & $\begin{array}{c}9.22 \\
(84.75)\end{array}$ & $\begin{array}{c}9.96 \\
(99.00)\end{array}$ & $\begin{array}{c}8.60 \\
(73.50)\end{array}$ & $\begin{array}{c}9.44 \\
(88.75)\end{array}$ \\
\hline $\begin{array}{l}\mathrm{W}_{2} \text { - Pendimethalin at } 1.0 \mathrm{~kg} \text { a.i. } \mathrm{ha}^{-1}(\mathrm{PE}) \mathrm{fb} \\
\text { Bispyribac at } 25 \mathrm{~g} \text { a.i. ha } \mathrm{h}^{-1}+\text { NIS }(0.25 \%) \text { at } 15-20 \\
\text { DAS }\end{array}$ & $\begin{array}{c}4.76 \\
(22.41)\end{array}$ & $\begin{array}{c}5.09 \\
(25.48)\end{array}$ & $\begin{array}{c}6.32 \\
(39.50)\end{array}$ & $\begin{array}{c}7.07 \\
(49.64)\end{array}$ & $\begin{array}{c}8.27 \\
(68.04)\end{array}$ & $\begin{array}{c}9.04 \\
(81.38)\end{array}$ & $\begin{array}{c}10.18 \\
(103.32)\end{array}$ & $\begin{array}{c}11.06 \\
(122.18)\end{array}$ & $\begin{array}{c}9.64 \\
(92.57)\end{array}$ & $\begin{array}{c}10.63 \\
(112.70)\end{array}$ \\
\hline $\begin{array}{l}\mathrm{W}_{3}-\text { Bispyribac at } 25 \mathrm{~g} \text { a.i. } \mathrm{ha}^{-1}+\text { Pyrazosulfuron } \\
\text { at } 20 \mathrm{~g} \text { a.i. } \mathrm{ha}^{-1}+\text { NIS }(0.25 \%) \text { at 15-20 DAS }\end{array}$ & $\begin{array}{c}5.04 \\
(24.72)\end{array}$ & $\begin{array}{c}5.44 \\
(29.16)\end{array}$ & $\begin{array}{c}6.00 \\
(35.56)\end{array}$ & $\begin{array}{c}6.79 \\
(45.88)\end{array}$ & $\begin{array}{c}8.04 \\
(64.30)\end{array}$ & $\begin{array}{c}8.80 \\
(77.02)\end{array}$ & $\begin{array}{c}9.93 \\
(98.25)\end{array}$ & $\begin{array}{c}10.76 \\
(115.70)\end{array}$ & $\begin{array}{c}9.37 \\
(87.40)\end{array}$ & $\begin{array}{c}10.34 \\
(106.64)\end{array}$ \\
\hline $\begin{array}{l}\mathrm{W}_{4^{-}} \text {Oxadiargyl at } 90 \mathrm{~g} \text { a.i. ha } \\
\text { at } 25 \mathrm{~g} \text { a.i. } \mathrm{ha}^{-1}+\mathrm{fb} \text { Bispyribac } \\
\text { al }(0.25 \%) \text { at } 15-20 \text { DAS }\end{array}$ & $\begin{array}{c}4.81 \\
(22.97)\end{array}$ & $\begin{array}{c}5.16 \\
(26.21)\end{array}$ & $\begin{array}{c}6.59 \\
(43.01)\end{array}$ & $\begin{array}{c}7.32 \\
(53.25)\end{array}$ & $\begin{array}{c}8.48 \\
(71.58)\end{array}$ & $\begin{array}{c}9.22 \\
(84.64)\end{array}$ & $\begin{array}{c}10.41 \\
(108.09)\end{array}$ & $\begin{array}{c}11.34 \\
(128.43)\end{array}$ & $\begin{array}{c}9.86 \\
(96.99)\end{array}$ & $\begin{array}{c}11.01 \\
(121.03)\end{array}$ \\
\hline $\begin{array}{l}\text { W }_{5^{-}} \text {Bispyribac at } 25 \mathrm{~g} \text { a.i. ha }{ }^{-1}+\text { Azimsulfuron at } \\
\left.17.5 \mathrm{~g}^{\text {a.i. }} \mathrm{ha}^{-1}\right)+ \text { NIS }(0.25 \%) \text { at 15-20 DAS }\end{array}$ & $\begin{array}{c}4.98 \\
(24.13)\end{array}$ & $\begin{array}{c}5.33 \\
(27.99)\end{array}$ & $\begin{array}{c}5.73 \\
(32.49)\end{array}$ & $\begin{array}{c}6.52 \\
(42.20)\end{array}$ & $\begin{array}{c}7.88 \\
(61.73)\end{array}$ & $\begin{array}{c}8.54 \\
(72.55)\end{array}$ & $\begin{array}{c}9.65 \\
(92.92)\end{array}$ & $\begin{array}{c}10.40 \\
(108.07)\end{array}$ & $\begin{array}{c}9.17 \\
(83.82)\end{array}$ & $\begin{array}{c}9.99 \\
(99.58)\end{array}$ \\
\hline SEm \pm & 0.063 & 0.061 & 0.080 & 0.077 & 0.087 & 0.109 & 0.100 & 0.119 & 0.103 & 0.109 \\
\hline $\mathrm{CD}(\mathrm{P}=0.05)$ & 0.179 & 0.174 & 0.228 & 0.221 & 0.247 & 0.312 & 0.284 & 0.339 & 0.295 & 0.313 \\
\hline
\end{tabular}


Table.2 Effect of nitrogen management and weed management practices on NPK removal $\left(\mathrm{kg} \mathrm{ha}^{-1}\right)$ of weed at 60 DAS in direct seeded rice

\begin{tabular}{|c|c|c|c|c|c|c|}
\hline \multirow[t]{2}{*}{ Treatments } & \multicolumn{2}{|c|}{ N removal $\left(\mathrm{kg} \mathrm{ha}^{-1}\right)$} & \multicolumn{2}{|c|}{ P removal $\left(\mathrm{kg} \mathrm{ha}^{-1}\right)$} & \multicolumn{2}{|c|}{ K removal (kg ha $\left.{ }^{-1}\right)$} \\
\hline & 2015 & 2016 & 2015 & 2016 & 2015 & 2016 \\
\hline \multicolumn{7}{|l|}{ Nitrogen management } \\
\hline $\mathbf{N}_{1^{-}}-1 / 2$ at basal $+1 / 4$ at active tillering stage $+1 / 4 \mathbf{N}$ at panicle initiation stage & 15.17 & 22.99 & 3.18 & 5.02 & 21.50 & 28.18 \\
\hline $\mathbf{N}_{2^{-}} 1 / 4 \mathbf{N}$ at basal $+1 / 2 \mathrm{~N}$ at active tillering stage $+1 / 4$ at panicle initiation stage & 17.51 & 26.40 & 4.06 & 6.60 & 23.31 & 31.09 \\
\hline $\begin{array}{l}\mathrm{N}_{3^{-}} 1 / 3 \mathrm{~N} \text { at basal }+1 / 3 \mathrm{~N} \text { at active tillering stage }+1 / 3 \mathrm{n} \text { at panicle initiation } \\
\text { stage }\end{array}$ & 13.32 & 19.79 & 2.18 & 3.61 & 19.50 & 24.46 \\
\hline $\begin{array}{l}\mathbf{N}_{4^{-}} 1 / 4 \mathrm{~N} \text { at basal }+1 / 4 \text { at active tillering stage }+1 / 4 \mathrm{~N} \text { at panicle initiation stage }+1 / 4 \\
\text { at heading stage }\end{array}$ & 11.84 & 18.18 & 2.03 & 3.25 & 18.29 & 22.29 \\
\hline SEm \pm & 0.384 & 0.527 & 0.064 & 0.105 & 0.613 & 0.653 \\
\hline $\mathrm{CD}(\mathbf{P}=\mathbf{0 . 0 5})$ & 1.328 & 1.824 & 0.216 & 0.364 & 2.121 & 2.259 \\
\hline \multicolumn{7}{|l|}{ Weed management practices } \\
\hline $\mathrm{W}_{0}$ - Weedy check & 25.15 & 37.81 & 6.34 & 9.99 & 31.21 & 43.86 \\
\hline $\mathrm{W}_{1^{-}}$Two hand weedings at 20 and 40 DAS & 9.51 & 13.56 & 1.28 & 2.15 & 15.01 & 17.54 \\
\hline $\begin{array}{l}\mathrm{W}_{2} \text { - Pendimethalin at } 1.0 \mathrm{~kg} \text { a.i. } \mathrm{ha}^{-1}(\mathrm{PE}) \text { fb Bispyribac at } 25 \mathrm{~g} \text { a.i. ha }{ }^{-1}+\mathrm{NIS} \\
(0.25 \%) \text { at } 15-20 \text { DAS }\end{array}$ & 13.51 & 20.83 & 2.56 & 4.13 & 20.03 & 25.17 \\
\hline $\begin{array}{l}\mathrm{W}_{3} \text { - Bispyribac at } 25 \mathrm{~g} \text { a.i. ha }{ }^{-1}+\text { Pyrazosulfuron at } 20 \mathrm{~g}^{\text {a.i. }} \mathrm{ha}^{-1}+\mathrm{NIS}(0.25 \%) \\
\text { at 15-20 DAS }\end{array}$ & 12.50 & 19.31 & 2.26 & 3.66 & 18.78 & 23.67 \\
\hline $\begin{array}{l}\mathrm{W}_{4^{-}} \text {Oxadiargyl at } 90 \mathrm{~g} \text { a.i. } \mathrm{ha}^{-1}(\mathrm{PE}) \mathrm{fb} \text { Bispyribac at } 25 \mathrm{~g} \text { a.i. } \mathrm{ha}^{-1}+\text { NIS }(0.25 \%) \\
\text { at 15-20 DAS }\end{array}$ & 14.67 & 22.30 & 2.92 & 4.66 & 21.20 & 27.03 \\
\hline $\begin{array}{l}\left.\text { W }_{5^{-}} \text {Bispyribac at } 25 \mathrm{~g} \text { a.i. } \mathrm{ha}^{-1}+\text { Azimsulfuron at } 17.5 \mathrm{~g} \text { a.i. } \mathrm{ha}^{-1}\right)+\mathrm{NIS}(0.25 \%) \\
\text { at 15-20 DAS }\end{array}$ & 11.43 & 17.21 & 1.80 & 3.10 & 17.68 & 21.78 \\
\hline $\operatorname{SEm} \pm$ & 0.400 & 0.550 & 0.062 & 0.100 & 0.650 & 0.683 \\
\hline $\mathrm{CD}(\mathrm{P}=\mathbf{0 . 0 5})$ & 1.144 & 1.571 & 0.179 & 0.285 & 1.858 & 1.953 \\
\hline
\end{tabular}


Table.3 Effect of nitrogen management and weed management practices on NPK removal $\left(\mathrm{kg} \mathrm{ha}^{-1}\right)$ of weed at Harvest in direct seeded rice

\begin{tabular}{|c|c|c|c|c|c|c|}
\hline \multirow[t]{2}{*}{ Treatments } & \multicolumn{2}{|c|}{ N removal $\left(\mathrm{kg} \mathrm{ha}^{-1}\right)$} & \multicolumn{2}{|c|}{ P removal $\left(\mathrm{kg} \mathrm{ha}^{-1}\right)$} & \multicolumn{2}{|c|}{ K removal (kg ha ${ }^{-1}$} \\
\hline & 2015 & 2016 & 2015 & 2016 & 2015 & 2016 \\
\hline \multicolumn{7}{|l|}{ Nitrogen management } \\
\hline $\mathbf{N}_{1}-1 / 2$ at basal $+1 / 4$ at active tillering stage $+1 / 4 \mathbf{N}$ at panicle initiation stage & 30.55 & 44.18 & 7.84 & 11.02 & 37.96 & 50.15 \\
\hline $\mathbf{N}_{2^{-}}-1 / 4 \mathbf{N}$ at basal $+1 / 2 \mathrm{~N}$ at active tillering stage $+1 / 4$ at panicle initiation stage & 35.46 & 51.33 & 9.86 & 15.64 & 41.22 & 54.43 \\
\hline $\mathrm{N}_{3^{-}}$1/3 $\mathrm{N}$ at basal $+1 / 3 \mathrm{~N}$ at active tillering stage $+1 / 3 \mathrm{n}$ at panicle initiation stage & 25.99 & 37.04 & 5.78 & 10.50 & 34.96 & 45.96 \\
\hline $\begin{array}{l}\mathbf{N}_{4^{-}} 1 / 4 \mathrm{~N} \text { at basal }+1 / 4 \text { at active tillering stage }+1 / 4 \mathrm{~N} \text { at panicle initiation stage }+1 / 4 \text { at } \\
\text { heading stage }\end{array}$ & 23.42 & 35.18 & 5.23 & 9.57 & 33.54 & 42.70 \\
\hline SEm \pm & 0.704 & 0.893 & 0.155 & 0.318 & 0.729 & 1.040 \\
\hline $\mathrm{CD}(\mathrm{P}=0.05)$ & 2.437 & 3.090 & 0.536 & 1.100 & 2.521 & 3.599 \\
\hline \multicolumn{7}{|l|}{ Weed management practices } \\
\hline $\mathrm{W}_{0}$ - Weedy check & 50.22 & 71.78 & 15.17 & 26.87 & 54.45 & 75.10 \\
\hline $\mathrm{W}_{1^{-}}$Two hand weedings at 20 and 40 DAS & 19.08 & 27.68 & 3.59 & 5.24 & 27.61 & 34.21 \\
\hline $\begin{array}{l}\mathrm{W}_{2} \text { - Pendimethalin at } 1.0 \mathrm{~kg} \text { a.i. } \mathrm{ha}^{-1}(\mathrm{PE}) \text { fb Bispyribac at } 25 \mathrm{~g}^{\text {a.i. }} \mathrm{ha}^{-1}+\mathrm{NIS}(0.25 \%) \text { at } \\
\text { 15-20 DAS }\end{array}$ & 27.26 & 39.64 & 6.44 & 9.37 & 35.86 & 45.74 \\
\hline $\begin{array}{l}\text { W }{ }_{3} \text { - Bispyribac at } 25 \mathrm{~g} \text { a.i. } \text { ha }^{-1}+\text { Pyrazosulfuron at } 20 \mathrm{~g} \text { a.i. } \mathrm{ha}^{-1}+\mathrm{NIS}(0.25 \%) \text { at } 15-20 \\
\text { DAS }\end{array}$ & 25.15 & 36.54 & 5.84 & 8.33 & 33.73 & 43.95 \\
\hline $\begin{array}{l}\text { W } 4^{-} \text {Oxadiargyl at } 90 \mathrm{~g} \text { a.i. } \mathrm{ha}^{-1}(\mathrm{PE}) \mathrm{fb} \text { Bispyribac at } 25 \mathrm{~g} \text { a.i. } \mathrm{ha}^{-1}+\mathrm{NIS}(0.25 \%) \text { at } 15-20 \\
\text { DAS }\end{array}$ & 29.66 & 44.14 & 7.28 & 13.58 & 37.75 & 50.89 \\
\hline $\begin{array}{l}\left.\text { W } 5^{-} \text {Bispyribac at } 25 \mathrm{~g} \text { a.i. } \text { ha }^{-1}+\text { Azimsulfuron at } 17.5 \mathrm{~g} \text { a.i. } \text { ha }^{-1}\right)+ \text { NIS }(0.25 \%) \text { at } 15-20 \\
\text { DAS }\end{array}$ & 22.49 & 31.80 & 4.63 & 6.70 & 32.06 & 39.98 \\
\hline SEm \pm & 0.699 & 0.839 & 0.144 & 0.335 & 0.759 & 0.982 \\
\hline $\mathrm{CD}(\mathrm{P}=0.05)$ & 1.997 & 2.398 & 0.412 & 0.956 & 2.168 & 2.806 \\
\hline
\end{tabular}


Application of oxadiargyl at $90 \mathrm{~g}$ a.i. $\mathrm{ha}^{-1}(\mathrm{PE})$ fb bispyribac at $25 \mathrm{~g}$ a.i. $\mathrm{ha}^{-1}+$ NIS $(0.25 \%)$ at 15-20 DAS treatment was the least effective in reducing the nitrogen removal by weeds during both the years. However, all the weed management treatments were significantly superior to weedy check in this respect during both the years at $60 \mathrm{DAS}$ and at harvest stage of direct seeded rice crop. Significantly reduced nutrient depletion by weeds as weed infestation and their dry matter accumulation in these treatments were significantly lower than other treatments. This fact is in conformity with the findings of Angiras and Rana (1999).

\section{Phosphorus removal $\left(\mathrm{Kg} \mathrm{ha}^{-1}\right)$}

Nitrogen treatments caused significant variation in phosphorus removal by weeds during both the years and $1 / 4 \mathrm{~N}$ at basal $+1 / 4 \mathrm{~N}$ at active tillering stage $+1 / 4 \mathrm{~N}$ at panicle initiation stage $+1 / 4 \mathrm{~N}$ at heading stage proved significant superiority in minimizing the phosphorus removal by weeds during both the years than the application of $1 / 2$ at basal $+1 / 4$ at active tillering stage $+1 / 4 \mathrm{~N}$ at panicle initiation stage and $1 / 4 \mathrm{~N}$ at basal $+1 / 2 \mathrm{~N}$ at active tillering stage $+1 / 4 \mathrm{~N}$ at panicle initiation stage. However, it was at par with the application of $1 / 3 \mathrm{~N}$ at basal $+1 / 3 \mathrm{~N}$ at active tillering stage $+1 / 3 \mathrm{~N}$ at panicle initiation stage at both the stages of crop growth during both the years.

Amongst weed management practices, bispyribac at $25 \mathrm{~g}$ a.i. $\mathrm{ha}^{-1}+$ azimsulfuron at $17.5 \mathrm{~g}$ a.i. $\mathrm{ha}^{-1}+$ NIS $(0.25 \%)$ at $15-20$ DAS was most effective in reducing the phosphorus removal by weeds next to the two hand weedings at 20 and 40 DAS and was significantly superior to rest of the weed management treatments during both the years. The next best treatment was the application of bispyribac at $25 \mathrm{~g}$ a.i. $\mathrm{ha}^{-1}+$ pyrazosulfuron at 20 g a.i. $\mathrm{ha}^{-1}+$ NIS $(0.25 \%)$ at $15-20$ DAS at
60 DAS and at harvest during both the years of study. The highest removal of phosphorus by weeds was recorded under the application of oxadiargyl at $90 \mathrm{~g}$ a.i. ha ${ }^{-1}$ (PE) fb bispyribac at $25 \mathrm{~g}$ a.i. $\mathrm{ha}^{-1}+\mathrm{NIS}(0.25 \%)$ at 15-20 DAS during both the years. However, all the weed management treatments had significantly lower removal of phosphorus by weeds as compared to weedy check during both the years of experimentation. Significantly reduced nutrient depletion by weeds as weed infestation and their dry matter accumulation in these treatments were significantly lower than other treatments. This fact is in conformity with the findings of Angiras and Rana (1999).

\section{Potassium removal $\left(\mathrm{Kg} \mathrm{ha}^{-1}\right)$}

The data revealed that amongst nitrogen management treatments, the minimum potassium removal was recorded with the application of $1 / 4 \mathrm{~N}$ at basal $+1 / 4$ at active tillering stage $+1 / 4 \mathrm{~N}$ at panicle initiation stage $+1 / 4$ at heading stage which was at par with the application of $1 / 3 \mathrm{~N}$ at basal $+1 / 3 \mathrm{~N}$ at active tillering stage $+1 / 3 \mathrm{~N}$ at panicle initiation stage and significantly lower than application of $1 / 2 \mathrm{~N}$ at basal $+1 / 4 \mathrm{~N}$ at active tillering stage $+1 / 4 \mathrm{~N}$ at panicle initiation stage and $1 / 4 \mathrm{~N}$ at basal $+1 / 2 \mathrm{~N}$ at active tillering stage $+1 / 4$ at panicle initiation stage during both the years of experimentation.

Among weed management treatments, application of bispyribac at $25 \mathrm{~g}$ a.i. $\mathrm{ha}^{-1}+$ azimsulfuron at $17.5 \mathrm{~g}$ a.i. $\mathrm{ha}^{-1}+$ NIS $(0.25$ $\%)$ at 15-20 DAS recorded minimum potassium removal by weed which was the second best after two hand weedings at 20 and 40 DAS treatment and significantly lower than the remaining herbicide application treatments during both the years. The maximum potassium removal was recorded with the application of oxadiargyl at $90 \mathrm{~g}$ a.i. $\mathrm{ha}^{-1} \mathrm{fb}$ bispyribac at $25 \mathrm{~g}$ a.i. $\mathrm{ha}^{-1}+$ NIS 
$(0.25 \%)$ at $15-20$ DAS. However, all the weed management treatments were significantly superior to weedy check in suppressing potassium removal by weeds during both the years at 60 DAS and at harvest stage of direct seeded rice. Significantly reduced nutrient depletion by weeds as weed infestation and their dry matter accumulation in these treatments were significantly lower than other treatments. This fact is in conformity with the findings of Angiras and Rana (1999).

Application of $1 / 4 \mathrm{~N}$ at basal $+1 / 4 \mathrm{~N}$ at active tillering stage $+1 / 4 \mathrm{~N}$ at panicle initiation stage $+1 / 4 \mathrm{~N}$ at heading stage and $1 / 3 \mathrm{~N}$ at basal + $1 / 3 \mathrm{~N}$ at active tillering stage $+1 / 3 \mathrm{~N}$ at panicle initiation stage were equally effective in minimizing weed dry weight and $\mathrm{N}, \mathrm{P}, \mathrm{K}$ depletion by weeds. Application of bispyribac at $25 \mathrm{~g}$ a.i. $\mathrm{ha}^{-1}+$ azimsulfuron at $17.5 \mathrm{~g}$ a.i. $\mathrm{ha}^{-1}+$ NIS $(0.25 \%)$ at 15-20 DAS and hand weeding twice at 20 and 40 DAS were recorded lower weed dryweight and nutrient depletion by weeds in direct seeded rice.

\section{References}

Amarjit, SB, Singh, M, Kachroo, D, Sharma, BC and Shrivan, DR, Efficacy of herbicides in transplanted medium duration rice (Oryza sativa) under subtropical conditions of Jammu, Indian Journal of Agronomy, 51(2), 128-130, 2006.

Angiras, NN and Rana, SS, Effect of safener doses and time of application of butachlor on weed control in direct seeded puddled rice (Oryza sativa L.),
Indian Journal of Weed Science, 30, (1/2), 21-24, 1999.

Chauhan, BS, Weed ecology and weed management strategies for dry seeded rice in Asia, Weed Technology, 26, 113, 2012.

Ghosh, D, Singh, UP, Brahmachari, K, Singh, NK and Das, A, An integrated approach to weed management practices in directseeded rice under zero tilled rice-wheat cropping system, International Journal of Pest Management, 63, (1), 37-46, 2017.

Joshi, E., Kumar, D., Lal, B., Nepalia, V., Gautam, P. and Vyas, A.K.2013.Management of direct seeded rice for enhanced resource - use efficiency. Pl. Knowledge J., 2: 119-34.

Mahajan, G, Chauhan, BS, Gill, MS, Dryseeded rice culture in Punjab state of India: lesson learned from farmers, Field Crops Research, 144, 89-99, 2013.

Maity, SK and Mukherjee, PK, Integrated weed management in dry direct-seeded rice (Oryza sativa). Indian Journal of Agronomy, 53, (2), 116-120, 2008.

Sanusan, S, Polthanee, A, Audebert, A, Seripong, $\mathrm{S}$ and Mouret, JC, Suppressing weeds in direct-seeded lowland rainfed rice: Effect of cutting dates and timing of fertilizer application, Crop Protection, 29, 927935, 2010.

Sweeney, AE, Renner, KA, Laboski, C and Davis, A, Effect of fertilizer nitrogen on weed emergence and growth, Weed Science, 56, 714-721, 2008.

\section{How to cite this article:}

Rama Devi B. and Yashwant Singh. 2018. Effect of Nitrogen and Weed Management on Nutrient Removal by Weed in Direct Seeded Rice. Int.J.Curr.Microbiol.App.Sci. 7(06): 14521459. doi: https://doi.org/10.20546/ijcmas.2018.706.172 